\title{
PARENTAL PRESENCE DURING INDUCTION OF ANESTHESIA: A SURVEY
}

\author{
Authors: Yuan-Chi Lin, MD, MPH, Raj Mehta, MD
}

Affiliations: Department of Anesthesiology, Perioperative and Pain Medicine, Children's Hospital Boston; Harvard Medical School, Boston, MA 02115, USA

INTRODUCTION: Parental presence during the induction of anesthesia can reduce the need for preoperative sedatives, and can decrease the child's anxiety during the separation to the operating room. This survey is designed to help us to understand the current practice of parental presence during the induction of anesthesia in major pediatric care centers in Northern America.

METHODS: Major pediatric care centers are identified through the National Association of Children's Hospital and Related Institute (NACHRI), Child Health Corporation of America (CHRA) and Accreditation Council for Graduate Medical Education (ACGME). We contacted the institutions by telephone and spoke with the operating room service anesthesiologists or preoperative care nurses. We inquired their institutional policy about allowing a parent to be present during the induction of anesthesia for pediatric patients. We made in two separate calls to each institution, by two separate investigators. If we found discrepancy in the results, a third phone call was made to determine the data for the final analysis.

RESULTS: We contacted 161 pediatric facilities, all of which are members of NACHRI or CHRA. Among those, 27\% are ACGME approved pediatric anesthesiology training programs. Overall $23 \%$ of the institutions allowed parental presence for the induction of the anesthesia. Interestingly, in comparing ACGME approved pediatric anesthesiology training programs with non-pediatric anesthesiology training facilities, the percentage of pediatric facilities allowing parents to be present during induction of anesthesia were $47 \%(20 / 43)$ vs. $14 \%$ (17/118), respectively, indicating a significantly higher rate of parental presence in ACGME approved facilities, based on Fisher' exact test $(\mathrm{P}<0.001)$.

DISCUSSION: There are advantages as well as disadvantages to parental presence during induction of anesthesia.. ${ }^{1,2,3,4,5}$ Our study indicates that parental presence during the induction of anesthesia is more commonly allowed in pediatric anesthesia ACGME approved pediatric anesthesiology training facilities than in non-ACGME approved pediatric anesthesiology teaching institutions.

\section{REFERENCES:}

1. An sth An ag. $200 \$ 6) 164 \$ 654$.

2. An eth बio b g.y200981)5864

3. An sth An ab. $2009 \$ 4) 97975$.

4. Can J An $\Theta$ th. 199 9; 8 74):1 7782

5. Can J An sth. 199 9; 14):2 715 